http://jmscr.igmpublication.org/home/ ISSN (e)-2347-176x ISSN (p) 2455-0450

crossref DOI: https://dx.doi.org/10.18535/jmscr/v7i10.08

\title{
Estimation of Time since Death by Histological Examination of Bowman Capsule in Human Kidney
}

\author{
Authors \\ Rajni Thakur', Anjana Tiwari ${ }^{2}$ \\ ${ }^{1}$ Assistant Professor, Department of Anatomy, Pt.J.N.M.Medical College, Raipur, (C.G.) \\ ${ }^{2}$ Associate Professor, Department of P.S.M, Govt. Medical College, Raigarh (C.G.)
}

\begin{abstract}
Background: All degenerative changes in Bowman's capsules of kidneys were influenced by duration, temperature, humidity. There are few studies on the estimation of time since death by histological examination of Bowman's capsules in human kidneys. This new study keen useful for every scientist that deals investigation on dead. The time of death estimation by histology of Bowman's capsules, plays important role in solving both criminal and civil cases.

Aim: Estimation of time since death by histological examination of Bowman's capsule.

Materials and Methods: This current research was carried out in Pt.J.N.M. Medical College and Dr. B.R. Ambedkar Memorial Hospital Raipur (C.G.), 40 cases sample from the kidneys of deceased, the Bowman's capsules were investigated to establish their correlation ship with hours PMI., temperatures, humidity or time since death by studied histological (H\& E, PAS staining). This research was done in between the temperature $17.3 / 22.3-31.3 / 45^{\circ}$ C, humidity $11 / 36$ to $75 / 95$ and duration range 4 hrs to $52.30 \mathrm{hr}$.

Result: In this research, the temperature $17.3 / 22.3-31.3 / 45^{\circ}$ C, humidity $11 / 36$ to $75 / 95$ and duration range 4hrs to 52.30hr.Bowman's capsule show progressive histological degenerative changes, at $52.30 \mathrm{hr}$, we found glomeruli-architecture disturbed disruption of epithelium with pyknotic nuclei, debris in the lumen. Substances were not seen. PAS staining -PAS +.

Conclusion: In this research, a significant linear correlation ship, at various degrees of degenerative changes, was seen to exist between PMI duration, temperature and humidity observed in Bowman's capsules, this kind of histological finding, help to reduce the error in estimating time since death.

Keywords: Bowman's capsule, criminal and civil, PMI, PAS staining, splitting.
\end{abstract}

\section{Introduction}

The kidney consists of an outer cortex and an inner medulla. The outer cortex of normal kidney contains the renal corpuscles which appear as large spherical structure and renal tubules. Each renal corpuscle is surrounded by the Bowman's capsule composed of simple squamous epithelial cells, composed of visceral and parietal epithelial separated by a potential space Bowman's space and the capillary tuft of the glumerulus which consists of blood capillaries and masengial cells ${ }^{1}$. Physiological time of death: The point at which the deceased's body - including vital organs ceased to function. Estimated time of death: A best guess based on available information. Legal time of death: The time at which the body was discovered or physically pronounced dead by another individual. This is the time that is shown - 
by law - on a death certificate ${ }^{2}$. It is very important from medico-legal point of view that a medical jurist should always be prepared to give an opinion as to the time which elapsed since death, when a body is brought to him for postmortem examination ${ }^{3}$.

Time since death is a question of day to day challenge faced by teams of investigators during their course of investigations. The physical signs require a definite experience and are subject to variations in interpretations from person to person, hence are extremely subjective in nature ${ }^{4}$. The point to be noted in ascertaining the time are warmth or cooling of the body, the absence or presence of cadaveric hypostasis, rigor mortis and the progress of decomposition. All these point discussed at full length in every Forensic Textbook, but it must be remembered that the conditions producing these changes vary so much in each individual case, that only very approximate time of death can be given ${ }^{5}$.

it was very crucial for all scientists had adequate knowledge to estimate accurately how long a person had been dead if they was to contribute to the resolution of the legal issues involved when a human body is recovered. The most common way to obtain such information is to conduct controlled studies on deceased individuals of known age, race, sex, weight, and cause manner of death in natural, rather than lab. All degenerative changes in Bowman's capsules of kidneys were influenced by duration, temperature, humidity. There are few studies on the estimation of time since death by histological examination of Bowman's capsules in human kidneys. This new study keen useful for every scientist that deals investigation on dead. The time of death estimation by histology of Bowman's capsules, plays important role in solving both criminal and civil cases.

\section{Methods}

The present research was carried out in Pt.J.N.M. Medical College and Dr. B.R. Ambedkar Memorial Hospital Raipur (C.G.). The dead bodies brought to the mortuary were used as material for collection of 40 cases sample from the kidneys of deceased. The Bowman's capsules were investigated to establish their correlation ship with hours PMI., temperatures, humidity or time since death by studied histological (H\& E, PAS staining). This research for which it was available was temperature between17.3/22.3$31.3 / 45^{\circ} \mathrm{C}$, humidity between $11 / 36$ to $75 / 95$ and duration range was between $4 \mathrm{hrs}$ to $52.30 \mathrm{hr}$. We included, the exact time of death of individual should be known not suffering from any disease affecting kidney. The exact time of death of individual should be known. We Excluded, unknown time of death, suffering from kidney disease, those preserve in ice or ice cooler, metabolic disorders.

\section{Observation and Results}

This research was shown that the rate of bodily decay is quite variable, general observations suggest that the three variables that most influence the rate of human bodily decay and histological finding was temperature, humidity, duration. The histological changes in Bowman's capsules occurring in the renal cortical area of human kidneys were obtained for investigation at temperature $17.3 / 22.3-31.3 / 45^{\circ} \mathrm{C}$, humidity $11 / 36$ to $75 / 95$ and duration range $4 \mathrm{hrs}$ to $52.30 \mathrm{hr}$. We observed the following post mortem histological changes in Bowman's capsules of human kidney, that's show progressive degenerative histological changes in Bowman's capsules. (Fig: 7).

Case no: 1- The post mortem interval (PMI)- 4 hrs, Temperature-27.5/42.2 ${ }^{0} \mathrm{C}$, humidity- $14 / 41 \%$, Bowman's capsules show glomeruli was expanded with contained dark stained nuclei and PAS staining - basement membrane of parietal layer of Bowman capsules was PAS+. (Fig: 1) \& (Fig: 8).

Case no: 2- PMI -5 hrs Temp- 18.1/34.6 ${ }^{0} \mathrm{C}$ humidity-20/42\%, glomeruli was expanded with dark stained nuclei.

Case no: 3- PMI 6hrs Temp- $18.5 / 33.3^{\circ} \mathrm{C}$ humidity-41/72\%, glomeruli was expanded with dark stained nuclei. 
Case no: 4- PMI 6.20 hrs Temp- $25.5 / 31^{0} \mathrm{C}$ humidity $85 / 87 \%$, glomeruli was slightly shrunk with dark stained nuclei.

Case no: 5- PMI 7.35 hrs Temp -26.8/37. ${ }^{\circ} \mathrm{C}$ humidity-41/71\%, glomeuli was expanded, having nuclei was dark stained mostly but few vesicular nuclei were also present.

Case no: 6- PMI 8.30hrs Temp- 9.8/26.3 ${ }^{0} \mathrm{C}$ humidity-33/65\%, glomeruli was expanded having vesicular, dark stained nuclei and red blood cells were present and PAS staining - basement membrane of Bowman's capsules was PAS+. (Fig: 6).

Case no: 7- PMI 12.30 hrs Temp- $24.5 / 38.1^{0} \mathrm{C}$ humidity-22/37, glomeruli was expanded, nuclei was vesicular as well as dark stained.

Case no: 8- PMI 12.30hrs Temp- $28.4 / 44.2^{0} \mathrm{C}$ humidity-16/27\%, glomeruli -expanded, with dark stained nuclei mostly, cells were edematous.

Case no: 9- PMI 13hrs Temp- $23.9 / 38.7^{0} \mathrm{C}$ humidity-31/70\%, glomeruli- expanded and disrupted with dark stained nuclei.

Case no: 10 - PMI 13hrs Temp- 23.9/38.7 ${ }^{0} \mathrm{C}$ humidity-41/72\%, glomeruli -expanded, with dark stained nuclei mostly.

Case no: 11 - PMI $13 \mathrm{hrs}$ Temp- $25.5 / 31^{0} \mathrm{C}$ humidity-85/87\%, glomeruli disrupted having dark stained nuclei.

Case no: 12 - PMI 13hrs Temp- 8.2/23.9 ${ }^{0} \mathrm{C}$ humidity-26/83\%, .glomeruli-expanded, disrupted with dark stained nuclei, red blood cells was present.

Case no: 13- PMI 13.10hrs Temp- 27.3/42.2 ${ }^{0} \mathrm{C}$ humidity-24/52\%, glomeruli- expanded with dark stained nuclei mostly, vesicular nuclei was seen.

Case no: 14- PMI 13.10hrs Temp- 27.3/42.2 ${ }^{0} \mathrm{C}$ humidity-24/52\%, glomeruli- expanded with dark stained nuclei.

Case no: 15 - PMI 13.30hrs Temp- 29.6/43.1 ${ }^{0} \mathrm{C}$ humidity-23/38\%, glomeruli- expanded with vesicular \& dark stained nuclei. PAS staining basement membrane of Bowman's capsules PAS+ completely. (Fig: 9).

Case no: 16- PMI 16hrs Temp- $11 / 23.7^{0} \mathrm{C}$ humidity-44/77\%, glomeruli -expanded, slightly fragmented having dark stained nuclei with somewhat pyknotic changes and vesicular nuclei were hardly seen. Bowman's space is increased with the presence of debris.

Case no: 17- PMI 16hrs Temp- $24.6 / 38.1^{0} \mathrm{C}$ humidity-36/46\%, glomeruli- splitted, slightly shrunk with dark stained nuclei mostly having pyknotic changes at places. Bowman's spaces were increased having debris

Case no: 18- PMI 16hrs Temp- 27.5/42.2 ${ }^{0} \mathrm{C}$ humidity-14/41\%, glomeruli- slightly shrunk with dark stained nuclei mostly. Bowman's spaces were increased having debris. (Fig: 2).

Case no: 19- PMI 16.30hrs Temp- $21 / 39^{\circ} \mathrm{C}$ humidity-11/36\%, glomeruli- splitted with dark stained nuclei. Bowman's spaces were increased with the presence of debris in the space.

Case no: 20- PMI 17hrs Temp- $12.8 / 23.5^{\circ} \mathrm{C}$ humidity-44/82\%, .glomeruli -shrunk mostly and splitted with dark stained nuclei having pyknotic changes at some places. Bowman's spaces were increased having debris.

Case no: 21- PMI 17.30hrs Temp- $27.3 / 42.2^{\circ} \mathrm{C}$ humidity-24/52\%, glomeruli- expanded with vesicular and dark stained nuclei.

Case no: 22- PMI 17.30hrs Temp- 27.5/42.2 ${ }^{\circ} \mathrm{C}$ humidity-14/41\%, glomeruli- shrunk and disruption of epithelium with dark stained nuclei. Bowman's spaces were increased having debris in the lumen.

Case no: 23- PMI 17.30hrs Temp- $18.6 / 28.2^{\circ} \mathrm{C}$ humidity-79/98\%, glomeruli- expanded and disrupted with dark stained nuclei. Bowman's spaces were increased having debris. PAS staining - basement membranes of Bowman capsules were PAS+.

Case no: 24 - PMI 19hrs Temp- $27.3 / 41.4^{0} \mathrm{C}$ humidity-18/37\%, glomeruli- expanded, dark stained nuclei present. Bowman's space was increased.

Case no: 25- PMI 19hrs Temp- $19.5 / 35^{\circ} \mathrm{C}$ humidity-18/35\%, glomeruli- expanded and disrupted with vesicular as well as dark stained nuclei. Bowman's spaces were increased. 
Case no: 26 - PMI 19.30hrs Temp- $31.3 / 45^{\circ} \mathrm{C}$ humidity-25/51\%, intestitium visible in the cortex, glomeruli- expanded, splitted with dark stained nuclei. Bowman's spaces are increased. PAS staining- basement membrane of Bowman capsules is completely PAS+.(Fig: 10).

Case no: 27- PMI 20hrs Temp- $25.5 / 31^{0} \mathrm{C}$ humidity-85/87\%, glomeruli- expanded with dark stained nuclei.

Case no: 28- PMI 20hrs Temp- $27.5 / 42.2^{0} \mathrm{C}$ humidity-14/41\%, glomeruli -shrunk and splitted with dark stained nuclei. Bowman's spaces were enlarged with presence of debris.

Case no: 29- PMI 20hrs Temp- 26.3/40.6 ${ }^{\circ} \mathrm{C}$ humidity-16/46\%, glomeruli with degenerative changes partially and rest having dark stained nuclei. Bowman's spaces were increased having debris.

Case no: 30- PMI 21hrs Temp- $25.5 / 31^{\circ} \mathrm{C}$ humidity-85/87\%, glomeruli- expanded and splitted with dark stained nuclei. Red blood cells were present in glomeruli.

Case no: 31- PMI $21 \mathrm{hrs}$ Temp- $25.5 / 31^{0} \mathrm{C}$ humidity-85/87\%, glomeruli - expanded with dark stained nuclei having pyknotic changes at places. Case no: 32- PMI 21hrs Temp-29.6/43.1 ${ }^{\circ} \mathrm{C}$ humidity-23/38\%,.glomeruli- expanded with dark stained nuclei at their periphery.

Case no: 33- PMI 21.30hrs Temp- $26 / 40.8^{0} \mathrm{C}$ humidity-45/50\%, glomeruli -shrunk with dark stained nuclei having pyknotic changes. Bowman's spaces were increased. PAS staining. Basement membrane of Bowman capsules is completely PAS+. (Fig: 11).

Case no: 34 - PMI 28hrs Temp- $23 / 33.4^{0} \mathrm{C}$ humidity-65/90\%, glomeruli expanded with dark stained nuclei. Bowman spaces were not visible.

Case no: 35- PMI 28hrs Temp- $26.7 / 38.7^{0} \mathrm{C}$ humidity-44/64\%, glomeruli- expanded having dark stained nuclei with pyknotic changes at few places; basement membranes of Bowman capsules were PAS+.

Case no: 36- PMI 40hrs Temp- $25.3 / 32.2^{0} \mathrm{C}$ humidity-68/87\%, intestitium prominent in the cortex, glomeruli -mostly expanded but shrunk at few places having dark stained nuclei with pyknotic changes at few places. Bowman, s spaces were increased.

Case no: 37- PMI 41hrs Temp- $24.3 / 25.9^{0} \mathrm{C}$ humidity-84/87\%, glomeruli were expanded as well as shrunk also at places, splitted having dark stained nuclei mostly with pyknotic changes at few places. Bowman's spaces were increased. (Fig: 3).

Case no: 38- PMI 45hrs Temp- 24.3/25.9 ${ }^{0} \mathrm{C}$ humidity-84/87\%, glomeruli-expanded and splitted having dark stained nuclei.

Case no: 39- PMI 46hrs Temp- 24.3/25.9 ${ }^{0} \mathrm{C}$ humidity-84/87\%, intestitium prominent in the cortex, glomeruli -splitted with degenerative changes in some places having dark stained nuclei with pyknotic changes at places. (Fig: 4).

Case no: 40- PMI 52.30hrs Temp- $24.5 / 32^{\circ} \mathrm{C}$ humidity-75/95\%, intestitium prominent in the cortex, glomeruli- architecture disturbed disruption of epithelium with pyknotic nuclei, debris in the lumen. PAS staining -PAS + substances were not seen. (Fig: 5).

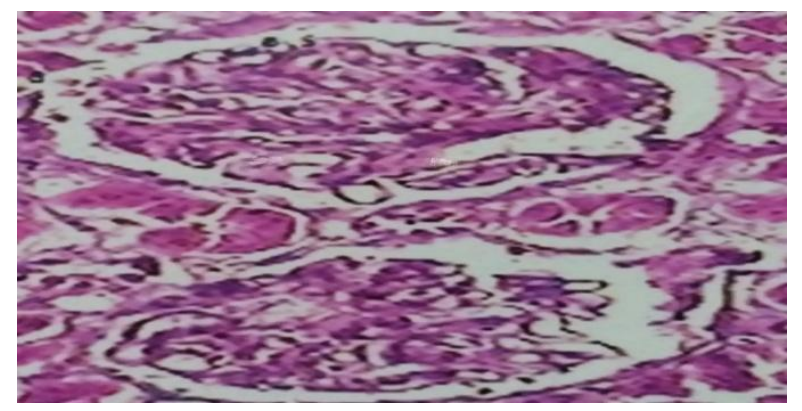

Fig: 1. 4hr Temp- $42.2 / 27.5^{\circ} \mathrm{C} \mathrm{H} \& \mathrm{E}$ stain 10X, Photomicrograph showing glomeruli (G) were expanded.

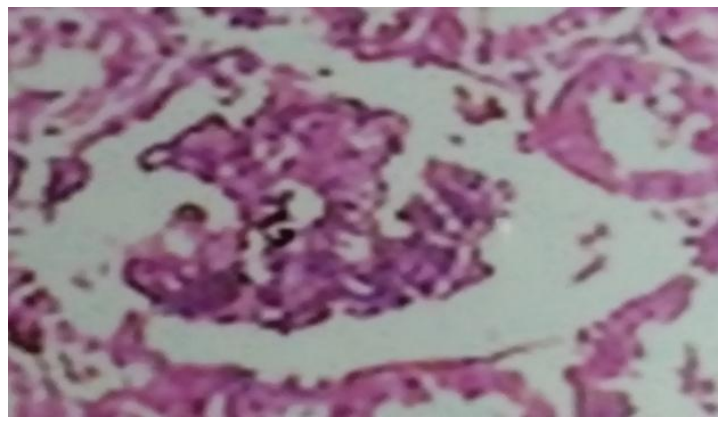

Fig: 2. $16 \mathrm{hrs}$ Temp27.5/42.2 ${ }^{\circ} \mathrm{C} \mathrm{H \& E}$ stain $10 \mathrm{X}$, Photomicrograph showing glomeruli $(\mathrm{G})$ shrunk; Bowman's spaces (B.S) were increased. 


\section{JMSCR Vol||07||Issue\|10||Page 41-48||October}

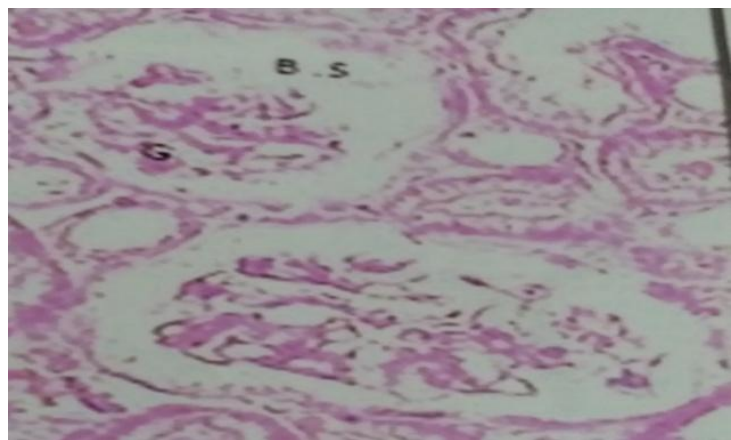

Fig: 3. $41 \mathrm{hr}$ Temp $24.3 / 25.9{ }^{\circ} \mathrm{C} \mathrm{H} \& \mathrm{E}$ stain $10 \mathrm{X}$, Photomicrograph showing glomeruli (G) were expanded as well as shrunk also at places, splitted, Bowman's spaces are increased.

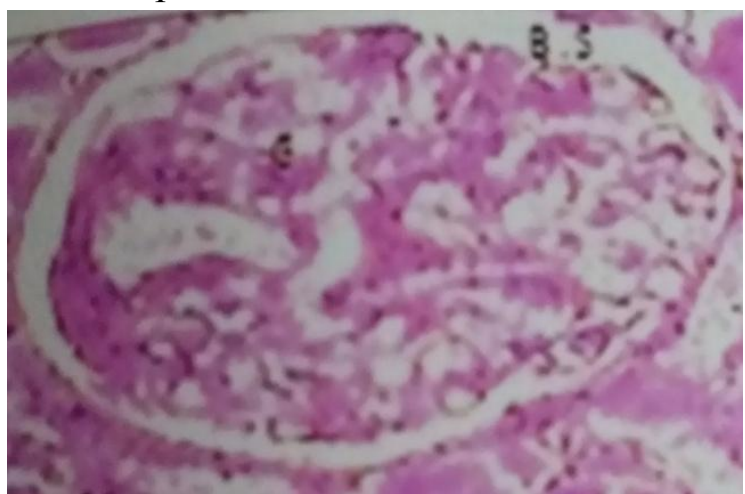

Fig: 4. 46 hr Temp $24.3 / 25.9^{\circ} \mathrm{C}$ H\&E stain 10X, Photomicrograph showing glomeruli (G) splited.

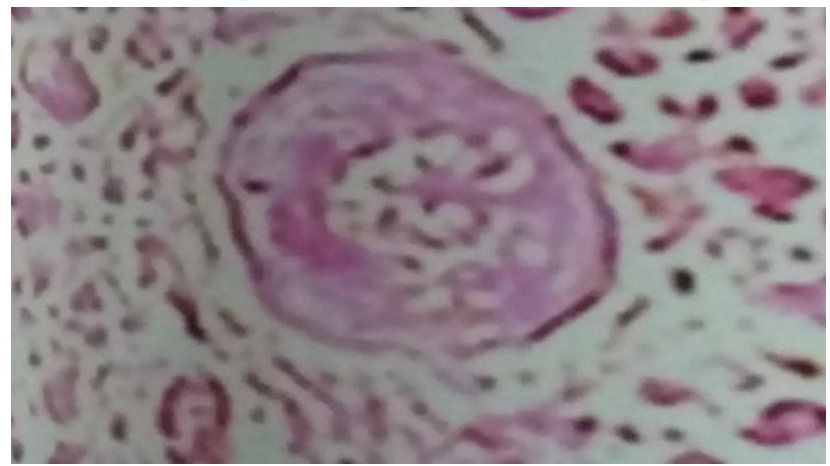

Fig: 5. $52.30 \mathrm{hr}$ Temp $32.0 / 24.5^{\circ} \mathrm{C} \mathrm{H \& E}$ stain 10X, Photomicrograph showing, intestitium prominent in the cortex, glomeruli(G) architecture disturbed.

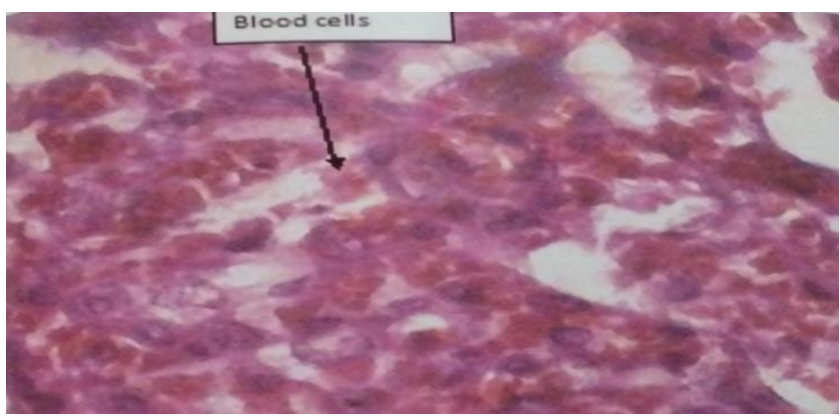

Fig: 6. $8.30 \mathrm{hr}$ Temp $-26.3 / 9.8^{\circ} \mathrm{C} \quad \mathrm{H} \& \mathrm{E}$ stain high power, Photomicrograph showing glomeruli (G) having red blood cells.

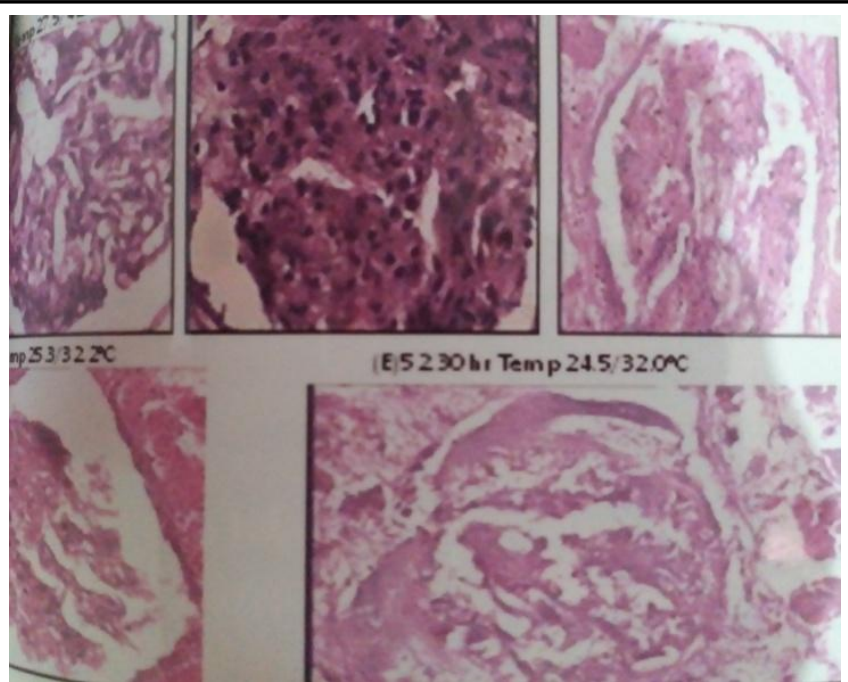

Fig: 7. H\&E stain high power photomicrograph showing (A) Glomeruli are expanded with dark stained nuclei(B) Glomeruli shrunk having dark stained nuclei with pyknotic changes, Bowman, space is increased (C) Glomeruli splitted with dark stained nuclei, pyknotic changes (D) Glomeruli splitted with dark stained nuclei having pyknotic changes at places. (E) Glomeruli architecture disturbed with pyknotic nuclei. Number of nuclei after 40 hours PMI temp $25.3 / 32.2^{0} \mathrm{C}(\mathrm{B})$ is less due to high temperature than the number of nuclei after $45 \mathrm{hr}$ PMI Temp $25.9 / 24.3{ }^{\circ} \mathrm{C}(\mathrm{C})$

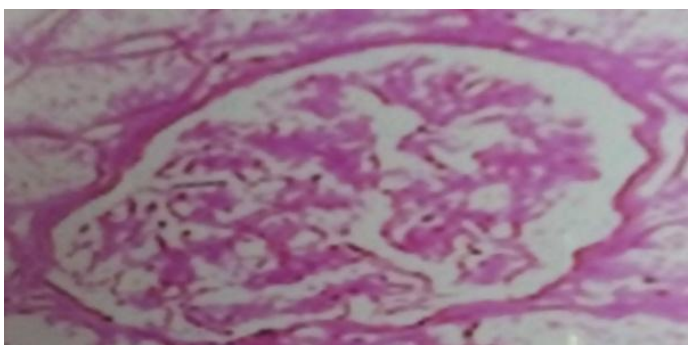

Fig.8. PAS staining PMI 4 hrs Temperature27.5/42. $2^{0} \mathrm{C}$ basement membrane of parietal layer of Bowman capsules were PAS+.

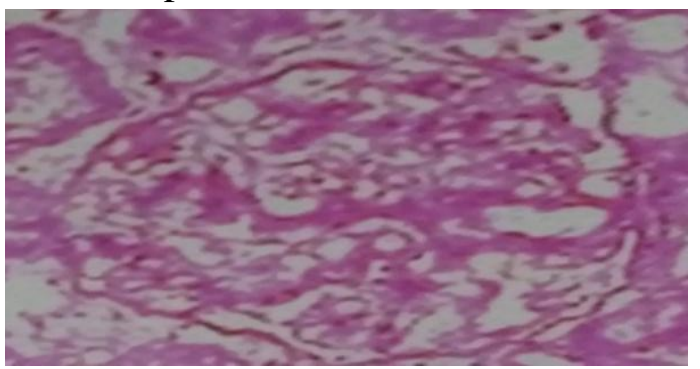

Fig.9. PAS staining PMI 13.30hrs Temp29.6/43.1 ${ }^{0} \mathrm{C} \quad$ humidity-23/38\% basement membrane of Bowman's capsules PAS+ completely. 


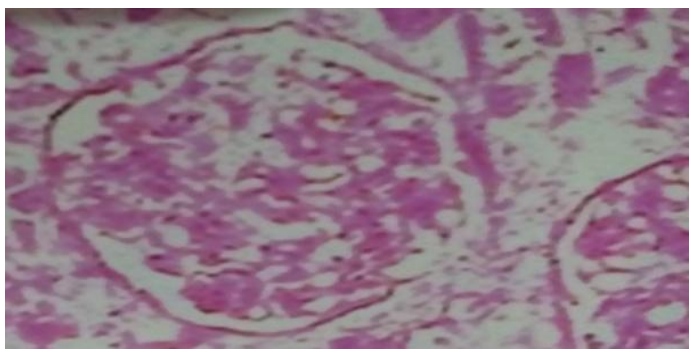

Fig.10. PAS staining PMI 19.30hrs Temp$31.3 / 45^{\circ} \mathrm{C}$ basement membrane of Bowman capsules is completely PAS+.

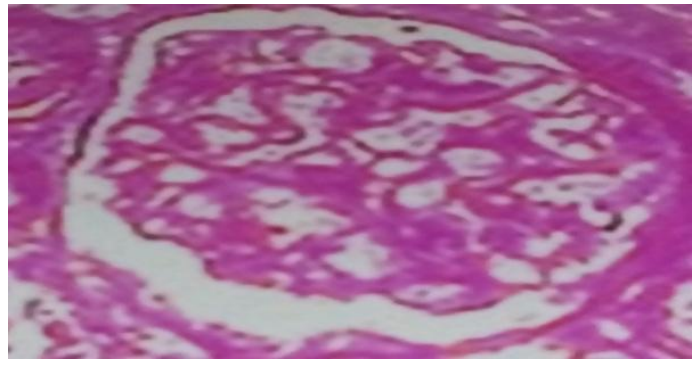

Fig.11. PAS staining PMI 21.30hrs Temp$26 / 40.8^{0} \mathrm{C}$ basement membrane of Bowman capsules is completely PAS+.

\section{Discussion}

Estimation of time passed since death continues to be a major problem for the forensic expert, anatomist, investigators scientists, Variety of physical and chemical postmortem changes were evaluated in past to estimate death interval but many of them become useful supplementary procedure. Survey of literature reveal that only few studies had been done to evaluate post mortem histological changes that occurred in human kidney after death to estimate time since death. In present research, postmortem histological changes in Bowman's capsules of kidney were observed in 40 cases of different age and sex. Average environmental temperature ranges between $17.3 / 22.3-31.3 / 45^{\circ} \mathrm{C}$,humidity between $11 / 36$ to $75 / 95$ and duration range was between $4 \mathrm{hrs}$ to $52.30 \mathrm{hrs}$. Degenerative changes were a continual process that can take from weeks to years, depending on the surrounding environment and the pivotal role is played by the deposition environment, and the climatic conditions, like temperature and humidity, as well as duration.
In our current research between, PMI- $4 \mathrm{hrs,}$ Temperature-27.5/42.2 ${ }^{0} \mathrm{C}$, humidity-14/41\% to 13 hrs Temp- $23.9 / 38.7^{\circ} \mathrm{C}$ humidity- $41 / 72 \%$, we found, glomeruli was expanded, slightly shrunk with dark stained nuclei cells as well as oedematous, few vesicular nuclei were also present. Red blood cells were present in some capsules and PAS staining - basement membrane of Bowman's capsules was PAS+. Kam at $\mathrm{el}^{6}$ found in cobra at $4 \mathrm{~h}, 8 \mathrm{~h}$ and $12 \mathrm{~h}$ time intervals, swelling of the epithelial cells lining, widening of their urinary spaces and congestion of glomeruli, cytoplasmic degeneration of epithelia, pyknosis of nuclei and compression of glomeruli. More drastic alterations were seen after $12 \mathrm{~h}$, glomeruli were greatly compressed and pyknosis was prominent in nuclei of mesangial and endothelial cells. Large aggregations of deeply stained nuclei present. Deborah Barber ${ }^{7}$ found in Porcine kidney, 3 Hours $24^{\circ} \mathrm{C}$ to 12 hours at $24^{\circ} \mathrm{C}$, glomeruli initially swelled resulting in narrowed or obliterated Bowman's spaces. This was due to fluid uptake by the cells. Bowman's space of some glomeruli was reduced or obliterated in all specimens, Ismail kati $^{8}$ found after $3 \mathrm{hrs}$ mild tubular atrophy, necrosis and fibrin deposition in the glomeruli. Vinita kushwaha et al. ${ }^{9}$ found in first 12 hrs (26$30^{\circ} \mathrm{C}$ T) showed mild degenerative changes (architecture maintained, mild cloudy swelling and disruption of tubular epithelium).

In our current research between, PMI 13hrs Temp- $25.5 / 31^{\circ} \mathrm{C}$ humidity- $85 / 87 \%$ to PMI $17 \mathrm{hrs}$ Temp- $12.8 / 23.5^{0} \mathrm{C}$ humidity-44/82\%, we found, glomeruli expanded, disrupted, slightly fragmented, splitted having vesicular dark stained nuclei, somewhat pyknotic changes at places, blood cells was present. Bowman's space is increased with the presence of debris. PAS staining - basement membrane of Bowman's capsules PAS+ completely. Vinita kushwaha et al ${ }^{9}$ found in their study after 13- $18 \mathrm{hrs}$ PMI with increasing temperature of up to 31 to $35^{\circ} \mathrm{C}$, moderate \& severe changes. Architecture maintained, more cloudy swelling and disruption of epithelium, glomeruli swollen. Only 2 out of 13 
cases showed severe changes, architecture disturbed, cloudy swelling and disruption of epithelium was prominent and collapse of glomeruli. Vineeta Girdoniya ${ }^{10}$ found glomerulus, pyknotic nuclei, epithelial necrosis, enlarged lumen of cavity.

In our current research between, PMI $17.30 \mathrm{hrs}$ Temp- $27.3 / 42.2^{0} \mathrm{C}$ humidity-24/52\% to PMI $21 \mathrm{hrs}$ Temp- $25.5 / 31^{\circ} \mathrm{C}$ humidity- $85 / 87 \%$, we found, intestitium visible in the cortex, glomeruliexpanded, shrunk and disruption, splitted of epithelium, red blood cells were present with vesicular and dark stained nuclei. Bowman's spaces were increased having debris in the lumen. PAS staining - basement membranes of Bowman capsules were PAS+. Khurshid Anwar ${ }^{11}$ found in newly hatch chicks kidney, reduction in the size of glomeruli as well as complete distortion of glomeruli.In some section accompanied by blood cell infiltration endothelial layer of Bowman's capsule and epithelial layer of glomeruli also showed sever damage. Vinita kushwaha et.al ${ }^{9}$. found in their study after 19- $24 \mathrm{hrs}$ PMI, with increasing temperature of up to 31 to $35^{\circ} \mathrm{C}$, severity increases, architecture disturbed, cloudy swelling and disruption of epithelium was prominent, collapse of glomeruli.

In our current research between, PMI $21 \mathrm{hrs}$ Temp- $25.5 / 31^{\circ} \mathrm{C}$ humidity- $85 / 87 \%$ to PMI 52.30hrs Temp- $24.5 / 32^{\circ} \mathrm{C}$ humidity-75/95\%, we found, intestitium prominent in the cortex, glomeruli - expanded; architecture disturbed disruption of epithelium, debris in the lumen. Bowman spaces were not visible with degenerative changes in most of the places having shrunk, splitted, dark stained nuclei having pyknotic changes at their periphery. Substances were not seen.PAS staining. Basement membrane of Bowman capsules is completely PAS+. Saif AlJammas $^{12}$ showed clear histological changes represented by glomerular atrophy and widening of Bowman's spaces. R. E. UCHEYA ${ }^{13}$ showed vascular congestion and glomeruli haemorrhage, while one of the maternal rats that had prolonged gestation period (44 days) with signs of abnormally high bleeding during parturition showed higher degree of kidney derangement which was evidenced by shrunken glomerulus's. Marcelo leite da Veiga ${ }^{14}$ found at $24 \mathrm{hrs}$ the kidney tissue showed an enlargement of intercapsular space with glomerular atrophy, hypertrophy of the kidney tubules, cells with small granules on its cytoplasm and little nuclear alteration, vacuoles in the cytoplasm. Vinita kushwaha et $\mathrm{al}^{9}$ found in their study only mild and moderate changes after 31-34hrsPMI with increasing temperature of up to 31 to $35^{\circ} \mathrm{C}$, collapse of glomeruli with maintained architecture. Ali Asghar ${ }^{15}$ found in Rat kidney, glomerular tuft increased in size and some place mesangial thickening with shrinkage of capillary tuft. In the present study after 40hrs PMI( $25.3 / 32.2^{0} \mathrm{C}, \mathrm{T}$ ) intestitium was prominent in the cortex. Glomeruli were mostly expanded and shrunk at few places with dark stained nuclei having psychotic changes at few places, Bowman,s spaces were increased. Retraction and disruption of epithelium with pyknotic nuclei. Deborah Barber $^{7}$ found in porcine kidney after 48 hours $\left(24^{0} \mathrm{C}\right)$ glomeruli contained pyknotic nuclei and debris within Bowman's space. The space was prominent due to shrinkage of the glomerular cellular components. Samar Omar Rabah ${ }^{16}$ found in mice kidney after $48 \mathrm{hrs}$ PMI dilated lumina and marked atropy of glomerular tufts was observed. Abhay Kumar Pandey ${ }^{17}$ found in Shrinkage of glomerular structure and increases in Bowman's space.

\section{Conclusion}

In this research, a significant linear correlation ship, at various degrees of degenerative changes, was seen to exist between PMI duration, temperature and humidity observed in Bowman's capsules, this kind of histological finding, and research in estimating post mortem interval so as to reduce the error in estimating time since death.

Conflict of Interest: None declared. 


\section{Acknowledgements}

The author was grateful to her adorable parents late Shree D.R.Mandavi, late Smt. Durga Mandavi and lovable brother late Shree Rajesh Mandavi for their blessings and inspirations. The authors are highly thankful to head of all three departments and their staff of Pt.J.N.M. Medical college Raipur, Chhattisgarh, India for their cooperation and support during the entire study period and also thankful to authors/ editors/publisher of all those articles journals which are cited and included in references of this manuscript.

\section{References}

1. El- Feki at el, (July. 2008) Modulatory effect of thymoquinone on the histological changes induced by imidacloprid in Albino Rats, J. Egypt. Soc. Toxicol. Vol. 39: 7984.

2. Jack Claridge, (19 Jan 2017)Estimating the Time of Death, Explore forensics, Updated.

3. Justice K Kannan at el, (2011) Modi's textbook of medical jurisprudence and toxicology, 24th ed,: 14; 354.

4. Biradar, G at el (2016) Estimation of time since death from cytoplasm changes of bone marrow cells. Journal of South India Medico legal Association, 8(2), 85-89.

5. Viras Patel et al ( June 2013) Estimation of time since death by gastric contents, Int J Cur Res Rev, Vol 05 (11) Page 133.

6. Kam, Hemm aid at el (2010) Histological and ultra structural, alterations in the the renal cortex of Rats induced by the Egyptian Cobra (Naja Haje) crude venom. J. Exp. Biol. (Zool.), 6(2): 319 - 330.

7. Deborah Barber (1980): Sequential Histological postmortem changes in porcine kidney and Adrenal glands, Department of Pathology, D.V.M.Kansas state University.

8. Ismail Kati (1940-1942): Histological studies on the kidney of Rats, Journal of
Animal and veterinal advances. Year 2010/Volume 9/issue 14/P.11.

9. Vinita Kushwaha et al.(2010):Time since death from degenerative changes in the kidney, J. Indian Academy of Forensic medicine 32 ((1).P.37.

10. Vineeta Girdoniya (February 2016) Study of Histological Changes in the Liver, Kidney, Gill and Muscles of Catla catla Exposed to Alkaline Pond Water ARJLS, Volume 2, Issue 2. 5-9..

11. Khurshid Anwar, Effect of permethrin treatment on the kidney of newly hatch chick, Pakistan Journal of applied Sciences 3 (5):P.317-330.

12. Saif Al-Jammas,(2009) Nephrotoxicity Induced by Cytosar in Rabbits Kidneys, American Journal of Medical and Biological Research, Vol. 7, No. 1, 1-5.

13. R. E. UCHEYA at el (2006) Histological changes in kidney structure following a long - term administration of paracetamol in pregnant Sprague Dawley Rats. Nigerian Journal of Physiological Sciences 21 (1-2):77-81.

14. Marcelo Leite da Veiga and et al (2002) Histopathologic changes in the kidney tissue of Proclilodus Lineatus Valenciennes. Brazilian archives of biology and technology in international journal Vol 45, N 2:p.171-175.

15. Ali Asgar (2011) Histological studies on the kidney of Rats, J Forensic Res: P; 160.

16. Samar Omar Rabah (2010) Acute taxol neprotoxicity: Histological and ultra structural studies of mice kidney parenchyma, Sardi Journal of biological Science 17, P.105-114.

17. Abhay Kumar Pandey at el (2015) Histological changes in Liver and Kidney of Cat fish heteroneustes fossilis exposed to penta chlorophenol, Plant Archives Vol. 15 No. 2, pp1117-1120. 